\title{
CONHECIMENTO E ATITUDES DE PACIENTES FRENTE A MEDIDAS PREVENTIVAS DO PÉ DIABÉTICO
}

\section{KNOWLEDGE AND ATTITUDES OF PATIENTS IN FRONT OF PREVENTIVE MEASURES OF DIABETIC FOOT}

\author{
Marilyse de Oliveira Meneses ${ }^{1} *$ Jaciane Santos Marques $^{2} *$ Aline Tavares Gomes $^{3} *$ Socorro $^{*}$ \\ Adriana de Sousa Meneses Brandão ${ }^{4} *$ Vinícius Alexandre da Silva Oliveira ${ }^{5} *$ Samira Rêgo $^{*}$ \\ Martins de Deus Leal ${ }^{6}$
}

\section{RESUMO}

OBJETIVO: Avaliar o conhecimento e atitudes de pacientes diabéticos frente a medidas preventivas do pé diabético em Teresina-PI. MÉTODO: Estudo com delineamento transversal, de caráter descritivo, com abordagem quantitativa, realizado com 84 usuários de uma Unidade Básica de Saúde, do município de Teresina-PI, capital do Estado do Piauí. Os dados foram coletados por meio de entrevista e analisados por meio de estatística descritiva, multivariada e de frequência. RESULTADOS: Verificou-se que 52,4\% ( $\mathrm{n}=44$ ) da amostra pontuou acima da mediana para a dimensão de atitudes de controle do diabetes mellitus (Med=21), ao passo que 58,3\% (n=49) apesentou pontuação superior para a dimensão de conhecimentos e atitudes sobre as medidas preventivas do pé diabético (Med=6). CONCLUSÃO: As informações coletadas apresentaram um grau significativo de déficit de conhecimento, indicando que os pacientes seguem as orientações de forma segmentada, desprendidas de um conhecimento prévio de seus potenciais riscos.

Palavras-chave: Diabetes Mellitus; Pé Diabético; Atitude; Conhecimento; Prevenção.

\section{ABSTRACT}

OBJECTIVE: To evaluate the knowledge and attitudes of diabetic patients regarding preventive measures for diabetic foot in Teresina-PI. METHOD: A cross-sectional, descriptive study with a quantitative approach, carried out with 84 users of a Basic Health Unit, in the city of Teresina-PI, capital of the State of Piauí. Data were collected through interviews and analyzed using descriptive, multivariate and frequency statistics. RESULTS: It was found that $52.4 \%(\mathrm{~N}=44)$ of the sample scored above the median for the dimension of attitudes to control diabetes mellitus $(\mathrm{Md}=21)$, while $58.3 \%(\mathrm{~N}=49)$ presented higher score for the dimension of knowledge and attitudes about preventive measures for diabetic foot $(\mathrm{Md}=6)$. CONCLUSION: The information collected showed a significant degree of knowledge deficit, indicating that patients follow the guidelines in a segmented way, detached from previous knowledge of their potential risks.

Keywords: Diabetes Mellitus; Diabetic Foot; Attitude; Knowledge; Prevention.

1 Graduada em Enfermagem. Pós-graduada em Saúde da Família e Comunidade pela Universidade Estadual do Piauí, TeresinaPiauí- Brasil. Mestranda em Enfermagem pelo Programa de Pós-Graduação em Enfermagem da Universidade Federal do Piauí, Teresina- Piauí- Brasil.

2 Graduada em Enfermagem. Pós-graduada em Saúde da Família e Comunidade pela Universidade Estadual do Piauí, TeresinaPiauí- Brasil. Mestranda em Enfermagem pelo Programa de Pós-Graduação em Enfermagem da Universidade Federal do Piauí, Teresina- Piauí- Brasil.

3 Graduada em Enfermagem. Pós-graduada em Saúde da Família e Comunidade pela Universidade Estadual do Piauí, TeresinaPiauí- Brasil. Mestranda em Enfermagem pelo Programa de Pós-Graduação em Ciências e Saúde da Universidade Federal do Piauí, Teresina- Piauí- Brasil.

${ }^{4}$ Graduada em Enfermagem. Mestra em Ciências e Saúde pela Universidade Federal do Piauí, Teresina- Piauí- Brasil. Professora efetiva da Universidade Estadual do Piauí, Picos- Piauí- Brasil.

${ }^{5}$ Graduado em Odontologia. Mestre em Políticas Públicas pela Universidade Federal do Piauí, Teresina- Piauí- Brasil. Doutorando em Epidemiologia pela Fundação Oswaldo Cruz (FIOCRUZ). Professor efetivo da Universidade Estadual do Piauí, Teresina- PiauíBrasil.

${ }^{6}$ Graduada em Enfermagem. Doutora em Farmacologia pela Universidade Federal do Ceará, Fortaleza- Ceará- Brasil. Professora efetiva da Universidade Estadual do Piauí, Teresina- Piauí- Brasil. 


\section{INTRODUÇÃO}

O Diabetes Mellitus (DM) consiste em um grupo de distúrbios metabólicos caracterizados por defeitos na síntese e/ou ação da insulina, o que gera um estado de hiperglicemia constante ${ }^{(1)}$. Este agravo é um dos principais problemas de saúde na contemporaneidade, em decorrência do número de afetados e de incapacitações, além da elevada mortalidade e dos elevados gastos relacionados com o seu tratamento e controle (2).

As complicações do DM se dividem em agudas e crônicas, sendo a hiperglicemia o fator primário no desencadeamento de tais. As complicações crônicas se dividem em microvasculares (englobando a nefropatia, retinopatia e neuropatia diabética) e macrovasculares, resultantes de alterações em grandes vasos, podendo acarretar infarto agudo do miocárdio, acidente vascular encefálico e doença vascular periférica ${ }^{(3-4)}$.

Entre as complicações vasculares do $\mathrm{DM}$, as úlceras em membros inferiores (popularmente denominado "pé diabético") definem-se como sendo infecção, ulceração e/ou destruição de tecidos moles associadas à doença arterial periférica e alterações neurológicas $^{(5)}$. As ulcerações provocam perda de mobilidade, diminuição da qualidade de vida e amputações de membros inferiores, representando, portanto, uma das mais devastadoras complicações crônicas da doença $^{(6)}$.

N DERME dos indivíduos com diabetes ${ }^{(7)}$ sendo as úlceras agentes precursores de $40 \%$ a $60 \%$ das amputações não traumáticas ${ }^{(6)}$ gerando ampla repercussão social e econômica. Em estudo realizado no estado de Sergipe, o custo hospitalar direto estimado no tratamento de pacientes internados por "pé diabético", desde a admissão até a alta ou óbito, variou de $\mathrm{R} \$ 943,72$ a $16.378,85$ por paciente, com média de $\mathrm{R} \$ 4.461,04$, enquanto que o valor de desembolso do Sistema Único de Saúde (SUS), variou de R \$ 96,95 a R \$ 2.410,18, por paciente, com média de $\mathrm{R} \$ 633,97^{(8)}$.

Diante disto, além das implicações econômicas para o sistema de saúde, as amputações em decorrência de ulcerações nos pés, provocam ampla repercussão no âmbito pessoal, familiar e socioeconômico, com comprometimento da autoimagem do indivíduo e perda da capacidade produtiva, o que certifica de forma inteligível a importância da implantação de medidas preventivas do "pé diabético" nos portadores de $\mathrm{DM}^{(9)}$.

Dentro desse contexto, o presente trabalho surge de um processo de territorialização que possibilitou o contanto direto com diversos pacientes que possuíam descompensação dos seus níveis glicêmicos, tendo provocado: úlceras em estágio avançado e amputação em consequência de 
complicações da doença.

A aproximação com esses usuários possibilitou reflexões sobre os impactos sociais e econômicos da doença, e suscitou o desejo de analisar, no âmbito da atenção primária, possíveis relações entre variáveis sociodemográficas/ socioeconômicas.

Assim, o objetivo geral é avaliar o conhecimento e atitudes de pacientes diabéticos frente a medidas preventivas do pé diabético em Teresina-PI.

\section{MÉTODO}

Trata-se de um estudo com delineamento transversal, de caráter descritivo e com abordagem quantitativa, realizado no período de março a julho de 2019, em uma Unidade Básica de Saúde (UBS), da regional Sul, do município de Teresina, capital do Estado do Piauí. A escolha por essa UBS se deu pelo fato de ser território de prática da Residência Multiprofissional em Saúde da Família e Comunidade (RMSFC), da Universidade Estadual do Piauí (UESPI).

Conforme dados fornecidos pelos 26 Agentes Comunitários de Saúde, de 5 equipes de Saúde da Família, verificou-se a existência de 510 pacientes diabéticos acompanhados pela referida UBS. Para atingir os objetivos propostos, utilizou-se um cálculo amostral para populações finitas, com erro amostral de $5 \%$, nível de confiança de $95 \%$ e prevalência presumida de $7 \%$ resultando em uma amostra de 84 pacientes. Destaca-se que o valor de prevalência presumida está em conformidade com estudo nacional ${ }^{(10)}$, que demonstrou a prevalência de Diabetes Mellitus no Brasil de 7,5\%, optando-se para o cálculo pelo emprego do valor de $7 \%$.

Os pacientes diabéticos foram selecionados de forma aleatória, tendo-se como critérios de inclusão pacientes com diagnóstico de Diabetes Mellitus, de ambos os sexos, com idade igual ou superior a 18 anos, acompanhados e cadastrados no sistema de Hipertensos e Diabéticos (HIPERDIA), das cinco equipes de Estratégia Saúde da Família (ESF) supracitadas, e que aceitarem assinar o Termo de Consentimento Livre e Esclarecido (TCLE).

Foram excluídos do estudo diabéticos já submetidos à amputação de ambos os pés, transtibial ou transfemoral, além de pacientes que em virtude de transtorno mental e/ou cognitivo estivessem impossibilitados de responder as perguntas objetivas do formulário.

Utilizou-se amostragem aleatória para seleção dos participantes, sendo os dados coletados por meio de visitas pré-agendadas com os agentes comunitários de saúdes aos participantes selecionados, mediante o uso de um Questionário Clínico e Sócio-demográfico / Socioeconômico e o Escore de Cosson.

O questionário Clínico e Sóciodemográfico / Socioeconômico, continha itens para avaliação do tempo de diagnóstico, 
tratamento medicamentoso utilizado,

Os dados foram tabulados e analisados comorbidades e complicações presentes, idade, sexo, estado civil, escolaridade, renda e ocupação. Para a realização da avaliação global de atitudes de controle ao Diabetes Mellitus e para avaliação do conhecimento sobre medidas preventivas do pé diabético utilizou-se o Escore de Cosson, com adaptações para adequação a pesquisa frente à realidade pesquisada e aos objetivos deste estudo.

O Escore de Cosson, por sua vez, foi desenvolvido por Cosson, Ney- Oliveira e Adan, em 2005 em uma pesquisa intitulada Avaliação do Conhecimento de Medidas Preventivas do Pé Diabético em Pacientes do Rio Branco, Acre ${ }^{(11)}$. O questionário de atitudes de controle ao Diabetes Mellitus é constituído por 15 itens, os quais englobam dados referentes a consultas médicas, tabagismo, etilismo, realização de exames para controlar a glicemia, dieta e atividade física. O questionário de conhecimentos e atitudes sobre medidas preventivas do pé diabético contempla 11 itens que abordam dados referentes a conhecimento sobre práticas preventivas no cuidado com os pés, a exemplo do corte correto das unhas, hidratação dos pés, uso de calçado adequado.

Ressalta-se que para o questionário de conhecimentos sobre medidas preventivas do pé diabético ocorreram adaptações resultando em 10 itens. 


\section{RESULTADOS}

Participaram da presente pesquisa 84 pacientes diabéticos acompanhados por equipes da Estratégia de Saúde da Família de uma Unidade Básica de Saúde no município de Teresina, Piauí. Com idades variando entre
27 e 94 anos (Média=67,6; DP=11,51), a maioria pertecente ao sexo feminino 63 (75\%), autodeclarada parda 54 (64,3\%), casada $31(36,9 \%)$ ou viúva $27(32,1 \%)$, com ensino fundamental incompleto 46 (54,8\%), renda entre 1 e 2 salários mínimos 48 $(57,1 \%)$, aposentadas $57(67,9 \%)$. (Tabela 1$)$.

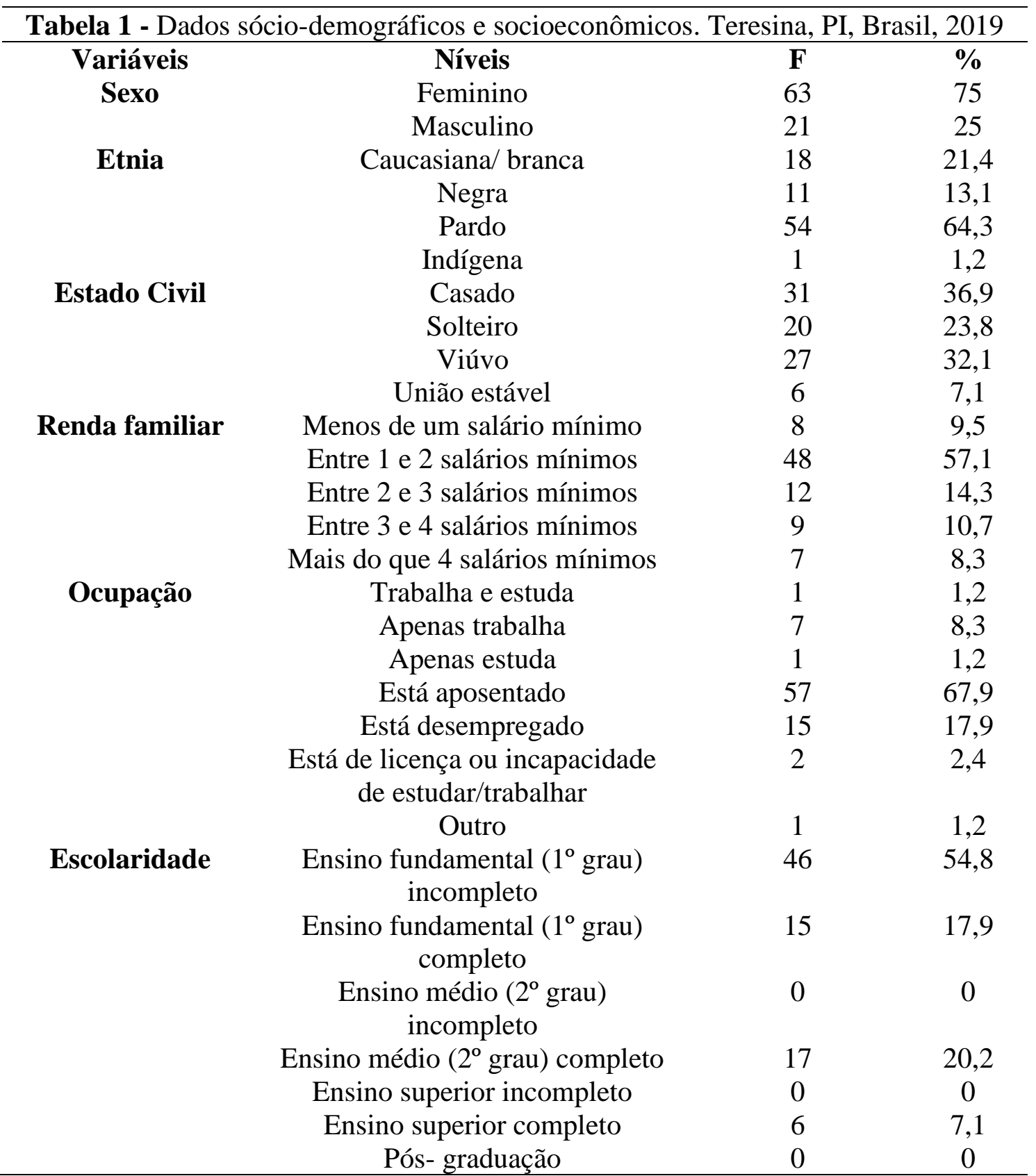

Fonte: Entrevista ofertada pelas depoentes (2019) 
No tocante aos dados clínicos, prevaleceu o diagnóstico de diabetes mellitus tipo 2, $81(96,4 \%)$, variando de 1 a 55 anos quanto ao tempo de diagnóstico ( $\mathrm{M}=11,7$; $\mathrm{DP}=10,4) ;$ o tipo de tratamento empregado em sua maioria corresponde aos antidiabéticos orais $63(75 \%)$. De modo adicional, em maior número, os participantes do estudo relataram não fazer parte de um grupo educativo voltado para a DM 81 $(96,4 \%)$. Quanto às comorbidades, a hipertensão arterial sistêmica aparece como o problema mais frequente relatado pelos participantes 66 (78,5\%), seguido de colesterol alto $47(55,9 \%)$, cardiopatia 27 $(32,1 \%)$ e retinopatia $25(29,7 \%)$. Salienta-se também que a maioria dos participantes identificou-se como ex- fumante 48 (57,1\%), relatou o não uso de bebida alcóolica 71 $(84,5 \%)$, realizar dieta alimentar $53(63,1 \%)$ e não praticar exercício físico 74 (88,1\%). (Tabela 2).

\begin{tabular}{|c|c|c|c|}
\hline \multicolumn{4}{|c|}{ Tabela 2- Dados Clínicos. Teresina, PI, Brasil, 2019} \\
\hline Variáveis & Níveis & $\mathbf{F}$ & $\%$ \\
\hline Diagnóstico de & Tipo 1 & 3 & 3,6 \\
\hline \multicolumn{4}{|l|}{ Diabetes Mellitus } \\
\hline \multirow{4}{*}{ Tratamento } & Tipo 2 & 81 & 96,4 \\
\hline & $\begin{array}{c}\text { Antidiabéticos orais } \\
\text { (ADO) }\end{array}$ & 63 & 75 \\
\hline & Insulina & 7 & 8,3 \\
\hline & $\begin{array}{c}\text { Associação ADO + } \\
\text { Insulina }\end{array}$ & 14 & 16,7 \\
\hline \multirow[t]{2}{*}{ Grupo educativo } & Sim & 3 & 3,6 \\
\hline & Não & 81 & 96,4 \\
\hline \multirow[t]{7}{*}{ Comorbidade } & $\begin{array}{l}\text { Hipertensão arterial } \\
\text { sistêmica }\end{array}$ & 66 & 78,5 \\
\hline & Obesidade & 17 & 20,2 \\
\hline & Colesterol alto & 47 & 55,9 \\
\hline & Doença renal & 17 & 20,2 \\
\hline & Cardiopatia & 27 & 32,1 \\
\hline & Retinopatia & 25 & 29,7 \\
\hline & Pé diabético & 14 & 16,6 \\
\hline \multirow[t]{3}{*}{ Tabagismo } & Não fumante & 31 & 36,9 \\
\hline & Fumante & 5 & 6 \\
\hline & Ex-fumante & 48 & 57,1 \\
\hline \multirow{2}{*}{$\begin{array}{l}\text { Uso de bebida } \\
\text { alcoólica }\end{array}$} & Sim & 13 & 15,5 \\
\hline & Não & 71 & 84,5 \\
\hline \multirow[t]{2}{*}{ Dieta alimentar } & Sim & 53 & 63,1 \\
\hline & Não & 32 & 36,9 \\
\hline \multirow[t]{2}{*}{ Exercício físico } & Sim & 10 & 88,1 \\
\hline & Não & 74 & 11,9 \\
\hline
\end{tabular}

Fonte: Entrevista ofertada pelas depoentes (2019) 
Concernente ao questionário de conhecimentos e atitudes sobre medidas preventivas do pé diabético, o mesmo mostrou que $69(82,1 \%)$ dos entrevisados não têm o hábito de andar descalço, apenas 17 $(20,2 \%)$ afirmaram ter o hábito de usar calçado fechado, $32(38,1 \%)$ usam meias quando calçam sapatos fechados, 54 (64,3\%) verificam o sapato antes de usar, 39 (46,4\%) secam os espaços interdigitais dos pés após o banho, apenas $72(85,7 \%)$ afirmaram não realizar escalda pés, $44(52,4 \%)$ realizam hidratação dos pés, $46(54,8 \%)$ realizam o corte reto das unhas, $61(72,6 \%)$ realizam a avaliação de calos, rachaduras e micoses nos pés diariamente, e por último, $43(51,2 \%)$ declaram possuir a pele do pé ressecada. Tais dados encontram-se representados nos Gráficos 01 e 02.

Gráfico 01- Escore de Cosson. Teresina, PI, Brasil, 2019

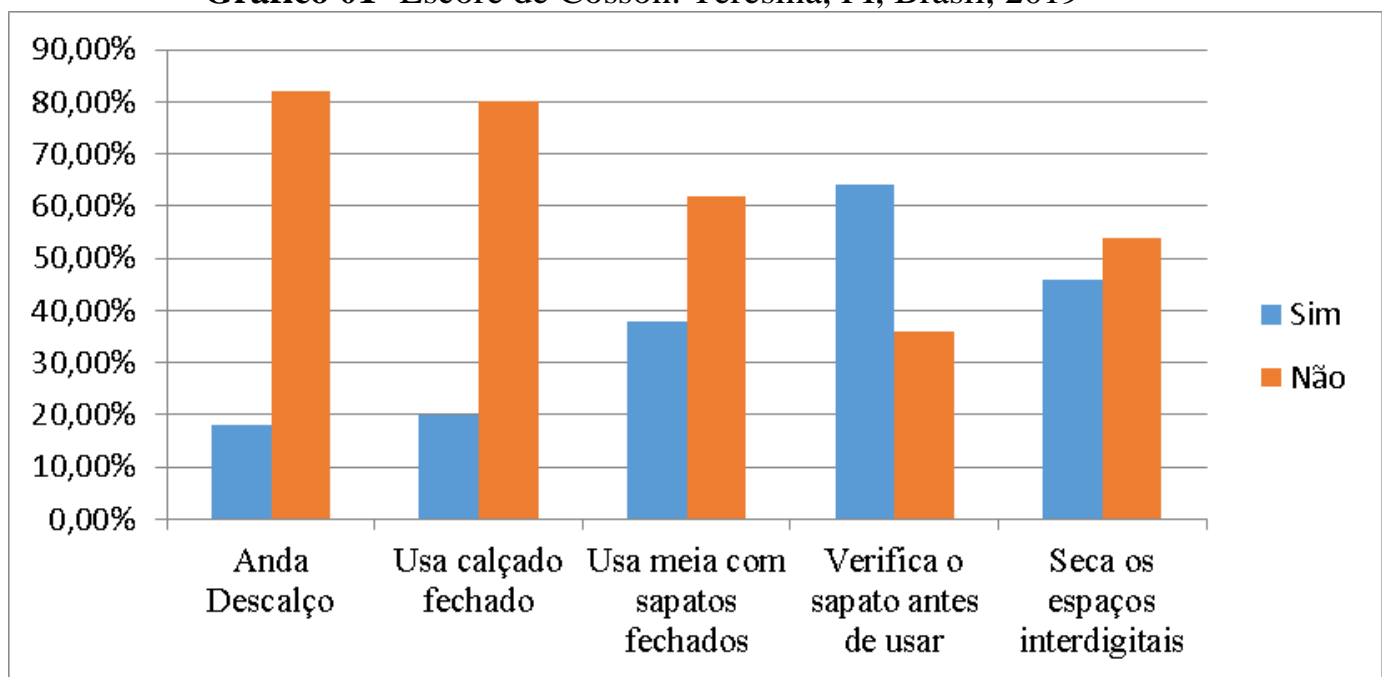

Fonte: Entrevista ofertada pelas depoentes (2019).

Gráfico 02- Escore de Cosson. Teresina, PI, Brasil, 2019

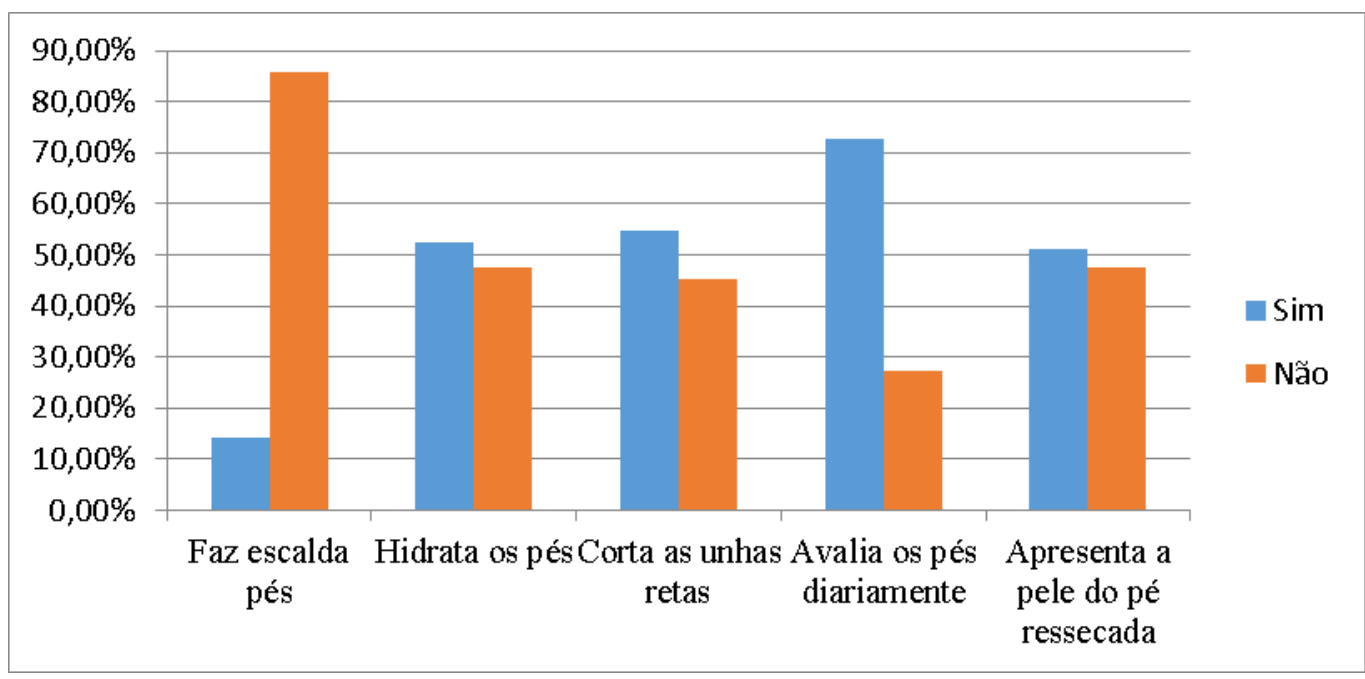


Fonte: Entrevista ofertada pelas depoentes (2019)

Considerando os objetivos do presente estudo, buscou-se avaliar também os escores de conhecimento dos pacientes diabéticos sobre medidas preventivas de cuidado com os pés, bem como a atitude dos pacientes diabéticos acerca do controle do Diabetes Mellitus. Para realizar essa avaliação, buscouse dividir os participantes considerando seus escores nas duas medidas utilizadas para avaliar essas dimensões. Os grupos foram divididos a partir da mediana empírica, ou seja, calculada a partir dos escores da própria amostra. Nessa direção, verificou-se que 44 $(52,4 \%)$ da amostra pontuou acima da mediana para a dimensão de atitudes de controle do diabetes mellitus $(\mathrm{Md}=21)$, ao passo que $49(58,3 \%)$ apresentou pontuação superior para a dimensão de conhecimentos e atitudes sobre as medidas preventivas do pé diabético $(\mathrm{Md}=6)$.

Acerca do sexo dos participantes; a análise não revelou diferenças significativas quanto ao conhecimento e a atitude frente ao pé diabético entre homens e mulheres [Lambda de Wilks $=0,98 ; \mathrm{F}(1,82)=0,69 ; \mathrm{p}$
$=0,50]$. Considerando como variável independente o estado civil dos participantes, também não se identificou diferenças estatísticamente significativas entre os grupos $[$ Lambda de Wilks $=0,95 ; \mathrm{F}(3,80)=0,57 ; \mathrm{p}$ $=0,75]$.

No tocante à renda familiar dos participantes em relação às variáveis dependentes; a investigação não revelou diferenças entre os grupos [Lambda de Wilks $=0,88 ; \mathrm{F}(4,79)=1,20 ; \mathrm{p}=0]$ por fim, investigou-se possíveis diferenças relacionadas ao grau de escolaridade dos participantes do estudo; a qual demonstrou não haver diferenças estatísticamente significativas entre os grupos [Lambda de Wilks $=0,90 ; \mathrm{F}(3,80)=1,43 ; \mathrm{p}=0,20]$.

De modo semelhante aos resultados oriundos das análises anteriores, buscou-se avaliar as relações entre idade e tempo de diagnóstico no tocante ao conhecimento e atitude frente ao pé diabético, onde também não foram identificadas relações estatisticamente significativas entre as variáveis de interesse. (Tabela 3).

Tabela 3- Correlação entre variáveis de interesse. Teresina, PI, Brasil,2019.

\begin{tabular}{|c|c|c|c|c|}
\hline & 1 & 2 & 3 & 4 \\
\hline Idade & 1 & & & \\
\hline Conhecimento & $-0,00$ & 1 & & \\
\hline Atitude & $-0,11$ & $0,35 * *$ & 1 & \\
\hline Tempo de diagnóstico & $-0,11$ & 0,13 & 0,15 & 1 \\
\hline
\end{tabular}

Fonte: Entrevista ofertada pelas depoentes (2019) 


\section{DISCUSSÃO}

Quanto aos dados clínicos, os resultados obtidos evidenciaram que o tempo de diagnóstico convergiu com os resultados apresentados em estudo com 235 pacientes com DM, onde $72,3 \%$ dos participantes afirmaram possuir o diagnóstico há mais de 10 anos. $^{(2)}$. Mediante isso, a literatura tem apontado que as complicações associadas à doença aumentam com um maior tempo de diagnóstico de DM e com a presença de comorbidades, sendo necessário promover estratégias que reduzam o surgimento de complicações prematuramente ${ }^{(12)}$.

Em relação as comorbidades, estudos semelhantes a este também demonstraram uma elavada porcentagem de participantes que afirmaram possuir diagnóstico de hipertensão arterial sistêmica. ${ }^{(2,}{ }^{13)}$. Com elevadas prevalências, a hipertensão arterial e o diabetes mellitus, destacam-se como potenciais causas de óbito na população idosa e principais fatores de risco para doenças cardiovasculares ${ }^{(4)}$.

Referente aos hábitos de vida relacionados ao uso do tabaco, os dados deste estudo foram inferiores aos encontrados em outra pesquisa, a qual demonstrou que $25 \%$ dos entrevistados identificaram-se como tabagistas ${ }^{(14)}$. No tocante ao hábito de ingerir bebida alccólica, os dados desta pesquisa, foram superiores ao encontrado em outro estudo, o qual revelaru que apenas $7 \%$ dos entrevistados, faziam uso de bebida alcoolica $^{(15)}$.

Com relação à realização de dieta alimentar e atividade física, os dados deste estudo apontaram que embora os participantes afirmassem realizar dieta alimentar, a quase totalidade dos mesmos, não realiza nenhum tipo de atividade física, dados que convergem com o estudo realizado com 64 indivíduos com diabetes mellitus tipo 2, na Paraíba, onde $76,5 \%$ dos entrevistados relataram fazer controle alimentar, mas apenas 25\% afirmaram realizar algum tipo de atividade física ${ }^{(14)}$.

Em relação aos cuidados com os pés, elevada porcentagem dos entrevisados afirmaram não ter o hábito de andar descalço, ficando na média de outro estudo que encontrou valor de $76,5 \%{ }^{(13)}$. Relativo ao hábito de usar calçado fechado, e ao uso de meias com sapatos fechados, estudo realizado em 30 Unidades de Saúde da Família, em Picos- Piauí demonstrou que apenas 12,7\% afirmou usar calçados fechados habitualmente, número inferior ao encontrado neste estudo ${ }^{(16)}$.

Contudo, relativo ao uso de meias com sapatos fechados, alguns estudos têm evidenciado números superiores, a exemplo do estudo realizado em Unidades Básicas de Saúde, em Campinas- $\mathrm{SP}^{(16)}$ e em pacientes frequentadores do Centro Integrado de Diabetes e Hipertensão, no estado do Ceará( ${ }^{(2)}$, https://doi.org/10.31011/reaid-2021-v.95-n.34-art.1034 Rev Enferm Atual In Derme v. 95, n. 34, 2021 e-021059 
os quais demonstraram respectivamente que $57 \%$ e $46,8 \%$ dos participantes fazem uso de meias com sapatos fechados.

Os calçados adequados são aqueles que suportam e protegem os pés contra traumas mecânicos, distribuindo os pontos de pressão, que não apresentam costuras e que estão em bom estado de conservação, não devendo ser muito largos, nem muito apertados, preferencialmente com meias de algodão devido ao risco de surgimento de bolhas ${ }^{(17)}$.

Relativo à secagem dos pés após o banho, embora $46,4 \%$ dos entrevistados tenham afirmado realizar a higienização e secagem dos espaços interdigitais, mais da metade dos participantes não faziam de maneira correta, deixando-os secar naturalmente ou realizando a secagem apenas do dorso, (estimulando a entrada de microorganismos). Resultado semelhante foi encontrado em outro estudo com 85 diabéticos, o qual evidenciou que menos da metade $45,9 \%$ reconhece que a secagem dos pés deve ser feita passando uma toalha por entre os dedos ${ }^{(16)}$.

Quanto ao hábito de realizar escalda pés, embora não prevalente entre os participantes da presente pesquisa, ainda é adotada por alguns indivíduos, conforme apontado por estudo transversal com 293 pessoas com DM2, o qual evidenciou que destes 105 ainda possuíam este hábito ${ }^{(13)}$, que se configura em hábito que provoca a perda de sensibilidade devido à neuropatia periférica.

Relativo à hidratação dos pés e ao corte reto das unhas - sem deixar pontas e, se necessário, lixando as unhas, os resultados desta pesquisa estão em acordo com estudo envolvendo 42 diabéticos, o qual demonstrou que $55 \%$ dos mesmos realizavam a hidratação dos pés e 52,5\% executavam o corte adequado das unhas ${ }^{(18)}$. Todavia, relativo ao corte adequado das unhas, estudo evidenciou que apenas $16,4 \%^{(15)}$.

No que tange as medianas dos escores de atitudes de controle do DM e de cuidados preventivos do pé diabético, convergindo com os achados nesta pesquisa, estudo semelhante verificou que na análise dos dados relativos ao conhecimento dos pacientes entrevistados em relação à prevenção do pé diabético, um número expressivo de pacientes possuía um conhecimento inadequado $(49,8 \%)^{(2)}$.

Os estudos nessa área indicam que no DM, o adequado nível instrucional consiste em fator de proteção imediatamente associado com o acesso e desfrute das informações e serviços de saúde, ao passo que, o baixo nível educacional pode prejudicar a compreensão e tratamento da doença, sendo considerado fator substancial para o controle glicêmico ${ }^{(11,19)}$.

Concernente a diferenças entre os grupos das variáveis clínicas e sociodemográficas e os escores de 
conhecimento e atitude frente ao pé diabético (variáveis dependentes), bem como as análises de correlação realizadas não identificaram diferenças estatisticamente significativas. Semelhantemente, outros estudos não conseguiram demonstrar diferenças de média entre as variáveis: sexo, escolaridade $^{(9)}$ e grau de instrução e idade ${ }^{(15)}$.

Os resultados apresentados, por sua vez, vão de encontro aos achados apresentados na literatura, a qual evidencia que a prevalência do sexo feminino, pessoas idosas e com baixa escolaridade podem influenciar na aceitação a terapia proposta, bem como a prevenção de agravos, sobretudo no que tange ao entendimento da doença e suas complicações ${ }^{(20)}$.

Mediante isso, espera-se que os resultados desta pesquisa fomentem novos conhecimentos científicos, auxiliem no desenvolvimento de novas pesquisas na área, além de contribuir com a implementação de ações educativas efetivas direcionadas ao desenvolvimento de competências para autocuidado, incluindo desde questões que envolvam a prática regular de atividade física até o aprendizado em torno da prevenção de úlceras nos pés.

\section{CONCLUSÃO}

Os resultados deste estudo apontaram para um grau significativo de déficit de conhecimento e atitudes frente a medidas preventivas do pé diabético, indicando que os pacientes seguem as orientações de forma segmentada, desprendidas de um conhecimento prévio de seus potenciais riscos. Dado o exposto, este estudo poderá contribuir com profissionais e serviços de saúde no desenvolvimento de intervenções eficazes voltadas para a gestão do autocuidado, prevenção do pé diabético e melhoria da qualidade de vida.

\section{REFERÊNCIAS}

1. Rossaneis MA, Andrade SM, Gvozd R, Pissinati PSC, Haddad MCL. Fatores associados ao controle glicêmico de pessoas com diabetes mellitus. Ciênc Saúde Colet [Internet]. 2019 [acesso em $10 \quad \mathrm{dez}$ 2018];24(3): 997-1005. Disponível em: https://www.scielo.br/pdf/csc/v24n3/1413-

8123-csc-24-03-0997.pdf.

2. Neto MO, Pereira MS, Pinto MAH, Agosto LM, Reinaldo Júnior FE, Hissa MN. Avaliação do autocuidado para a prevenção do pé diabético e exame clínico dos pés em um centro de referência em diabetes mellitus. J Health Biol Sci (Online) [Internet]. 2017 [acesso em 10 dez 2018];5(3):265-271. Disponível em: http://dx.doi.org/10.12662/23173076jhbs.v5i3.1092.p265-271.2017. 
3. Cecilio HPM, Arruda GO, Teston EF,

Santos AL, Marcon SS. Comportamentos e comorbidades associados as complicações microvasculares do diabetes. Acta Paul Enferm (Online). [Internet]. 2015 [acesso em 10 dez 2018];28(3):113-119. Disponível em: http://dx.doi.org/10.1590/1982-

0194201500020.

4. Sociedade brasileira de diabetes (SBD). Diretrizes da Sociedade Brasileira de Diabetes (2017-2018) [Internet]. São Paulo: A.C. Farmacêutica, 2017 [acesso em $11 \mathrm{dez} 2018$ ]. Disponível em: https://www.diabetes.org.br/profissionais/ima ges/2017/diretrizes/diretrizes-sbd-20172018.pdf.

5. Grupo de trabalho internacional sobre pé diabético. Consenso internacional sobre pé diabético [Internet]. Brasília: Secretaria de Estado de Saúde do Distrito Federal; 2001 [acesso em 11 dez 2018]. Disponível em: http://189.28.128.100/dab/docs/publicacoes/g eral/conce_inter_pediabetico.pdf.

\section{Santos ICRV, Sobreira CMM, Nunes ENS,} Morais MCA.Prevalência e fatores associados a amputações por pé diabético. Ciênc Saúde Colet [Internet]. 2013 [acesso em $11 \mathrm{dez}$ 2018];18(10):3007-14.Disponível em: http://dx.doi.org/10.1590/S141381232013001000025.
7. Pedra S, Carvalho R, Pereira MG. Sociodemographic and clinical characteristics of patients with diabetic foot ulcer. AMB rev Assoc Med Bras [Internet]. 2016 [acesso em 17 dez 2018];62(2):171-8. DOI: http://dx.doi.org/10.1590/18069282.62.02.171

8. Rezende KF, Nunes MAP, Melo NH, Malerbi D, Chacra AR, Ferraz MB. Internações por pé diabético: comparação entre o custo direto estimado e o desembolso do SUS. Arch endocrinol metab (Online) [Internet]. 2008 [acesso em 13 dez 2017];52(3): 523-530.Disponível em: http://dx.doi.org/10.1590/S000427302008000300013

9. Caiafa JS, Castro AA, Fidelis C, Santos VP, Silva ES, Sitrângulo Jr CJ. Atenção integral ao portador de pé diabético. $\mathbf{J}$ vasc bras [Internet]. 2011 [acesso em $13 \mathrm{dez}$ 2017];10(4 suppl 2):1-32. Disponível em: http://dx.doi.org/10.1590/S167754492011000600001.

10. Flor LS, Campos MR. Prevalência de diabetes mellitus e fatores associados na população adulta brasileira: evidência de um inquérito de base populacional. Rev bras epidemiol [Internet]. 2017 [acesso em $11 \mathrm{dez}$ 2018];20(1):16-29. Disponível em: http://dx.doi.org/10.1590/1980- 
5497201700010002.

11. Cosson ICO, Ney- Oliveira F, Adan LF. Avaliação do conhecimento de medidas preventivas do pé diabético em pacientes de Rio Branco, Acre.Arch endocrinol metab (Online) [Internet]. 2005 [acesso em $13 \mathrm{dez}$ 2018];49(4): 548-556. Disponível em: http://dx.doi.org/10.1590/S0004-

27302005000400013.

12. Cortez DN, Reis IA, Souza DAS, Macedo MML, Torres HC. Complicações e o tempo de diagnóstico do diabetes mellitus na atenção primária. Acta Paul Enferm (Online) [Internet]. 2015 [acesso em $13 \mathrm{dez}$ 2018];28(3): 250-5. Disponível em: http://dx.doi.org/10.1590/1982-

0194201500042.

13. Silva JMTS, Haddad MCFL, Rossaneis MA, Vannuchi, MTO, Marcon SS. Fatores associados à ulceração nos pés de pessoas com diabetes mellitus residentes em área rural. Rev gaúch enferm [Internet]. 2017 [acesso em 10 dez 2017];38(3):e68767. Disponível em: http://dx.doi.org/10.1590/19831447.2017.03.68767.

14. Mangueira HT, Silva ES, Oliveira CDB, Nascimento MBG, Félix TGS, Oliveira RR, Batista JLFP. Perfil epideomiológico de pacientes portadores de diabetes mellitus https://doi.org/10.31011/reaid-2021-v.95-n.34-art.1034 Rev Enferm Atual In Derme v. 95, n. 34, 2021 e-021059 cadastrados na atenção primária. Revista Enfermagem Atual In Derme [Internet]. 2020 [acesso em 4 abr 2021]; 94(32): e-20076. Disponível em: https://revistaenfermagematual.com/index.ph $\mathrm{p} /$ revista/article/view/775

15. Bragança CM, Gomes IC, Fonseca MRCC, Melissa NSC, Vieira MG, Souza MFM. Avaliação das práticas preventivas do pé diabético. J Health Sci Inst [internet]. 2010 [acesso em 13 dez 2018];28(2):15963.Disponível em: https://unip.br/presencial/comunicacao/public acoes/ics/edicoes/2010/02_abrjun/V28_n2_2010_p159-164.pdf.

16. Policarpo NS, Moura JRA, Melo Júnior EB, Almeida PC, Macêdo SF, Silva ARV. Conhecimento, atitudes e práticas de medidas preventivas sobre pé diabético. Rev gaúch enferm [Internet]. 2014 [acesso em $10 \mathrm{dez}$ 2018];35(3):36-42. Disponível em: https://doi.org/10.1590/19831447.2014.03.45187.

17. Grossi S. Prevenção de úlceras nos membros inferiores em pacientes com diabetes mellitus. Rev Esc Enferm USP [Internet]. 1998 [acesso em $10 \quad \mathrm{dez}$ 2018];32(4):377-85. Disponível em: https://doi.org/10.1590/S008062341998000400011. 
18. Cubas MR, Santos, OM, Retzlaff, EMA, Telma, HLC, Andrade, IPS, Moser, ADL, Erzinger, AR. Pé diabético: orientações e conhecimento sobre cuidados preventivos. Fisioter Mov (Online) [Internet]. 2013 [acesso em $13 \mathrm{dez}$ 2018];26(3): 647655.Disponível em: https://doi.org/10.1590/S010351502013000300019.

19. Hu J, Gruber KJ, Liu H, Zhao H, Garcia AA. Diabetes knowledge among older adults with diabetes in Beijing, China. $\mathrm{J}$ clin nurs [Internet]. 2013 [acesso em $20 \mathrm{dez}$ 2018]; 22(1-2):51-60. Disponível em: https://doi.org/10.1111/j.13652702.2012.04273.x.

20. Carolino IDR, Molena- Fernandes CA, Tasca RS, Marcon SS, CumanRKN. Fatores de risco em pacientes com diabetes mellitus tipo 2. Rev latinoam enferm (Online). [Internet]. 2008 [acesso em 11 dez 2018];16(2):238-244. Disponível em: https://doi.org/10.1590/S010411692008000200011.

Submissão: 2021-02-21

Aprovado: 2021-04-10 